\title{
Acne: prevalence, perceptions and beliefs among pupils and students in Ouagadougou, Burkina Faso
}

\begin{abstract}
Amina Nomtondo Ouedraogo,,2, Sandrine Soutongo Sita Kabre Ouedraogo ${ }^{3}$, Muriel Sidnoma Ouedraogo ${ }^{2,3}$, Fagnima Traore ${ }^{4}$, Patrice Gilbert Tapsoba ${ }^{2,3}$, Fatou Barro-Traore ${ }^{2}$, Adama Traore ${ }^{2,3}$, Pascal Niamba ${ }^{2,3}$
\end{abstract}

\begin{abstract}
${ }^{1}$ Department of Dermatology, Raoul Follereau Dermatology Center, Ouagadougou, Burkina Faso, ${ }^{2}$ University Ouaga I Professor Joseph Ki-Zerbo, Ouagadougou, Burkina Faso, ${ }^{3}$ Department of Dermatology, University Hospital Yalgado Ouédraogo, Ouagadougou, Burkina Faso, ${ }^{4}$ Department of Dermatology, Regional Hospital of Ouahigouya, Ouahigouya, Burkina Faso
\end{abstract}

Corresponding author: Dr. Amina Nomtondo Ouedraogo, E-mail: Nomtondo2000@yahoo.fr

\begin{abstract}
Introduction: Acne, is a common disease. Its prevalence is $80 \%$ among adolescents in the world and $57,1 \%$ in Ouagadougou in 2001 among school pupils. The medical literature is provided on the epidemiological, clinical and therapeutic aspects of acne, and less on perceptions and knowledge of acne patients of their disease. The objective of this study was to evaluate the prevalence, knowledge, beliefs on acne, among pupils and students in Ouagadougou. Patients and Methods: We conducted a cross-sectional study from June to July 2014 in the University of Ouagadougou and in 4 secondary schools in Ouagadougou. Results: We surveyed 425 personnes including $40.6 \%$ of girls and $59.4 \%$ boys. The prevalence of acne was $54.8 \%$. The mean age was 19.53 years, with extremes ranging from 13 to 30 years. Among the etiologies, food was cited by $40.05 \%, 25.43 \%$ puberty, cosmetics $14.03 \% 3.21 \%$ hormones, stress $2.33 \%$, $0.87 \%$ seborrhea. Among the foods concerned, peanuts were cited by $37.96 \%, 21.22 \%$ mayonnaise, butter, $21.52 \%$, $8.07 \%$ sweets and chocolate $9,41 \%$. oil was incriminated by $72.6 \%$ and sugar $5.5 \%$. Among the contributing factors, $19.70 \%$ cited tobacco, $19 \%$ no sexual relationship. Among the 192 non acne patients, $17 \%$ link acne to poor hygiene, $80 \%$ would be embarrassed to have a partner acne, $74.40 \%$ of non-acne believed that acne altered self-image. For treating acne $26.11 \%$ think that acne is incurable. Cosmetic can care acné according to 37,90\% and 6,30\% believed that natural products are sufficient. Only 15.02\% have resorted to a dermatologist. Conclusion: Pupils and students have bad knowledge on acne and bad perception on those who have the disease.
\end{abstract}

Key words: Diet; Acne etiology; Knowledge

\footnotetext{
How to cite this article: Ouedraogo AN, Kabre Ouedraogo SSS, Ouedraogo MS, Traore F, Tapsoba PG, Barro-Traore F, Traore A, Niamba P. Acne: prevalence, perceptions and beliefs among pupils and students in Ouagadougou, Burkina Faso. Our Dermatol Online. 2017;8(1):10-16.

Submission: 13.10.2016; Acceptance: 21.11.2016

DOI: 10.7241 /ourd.20171.03
} 


\title{
Acné: prévalence, perceptions et croyances chez les élèves et étudiants à Ouagadougou, Burkina Faso
}

\author{
Amina Nomtondo Ouedraogo, ${ }^{1,2}$, Sandrine Soutongo Sita Kabre Ouedraogo ${ }^{3}$, \\ Muriel Sidnoma Ouedraogo ${ }^{2,3}$, Fagnima Traore ${ }^{4}$, Patrice Gilbert Tapsoba ${ }^{2,3}$, \\ Fatou Barro-Traore ${ }^{2}$, Adama Traore ${ }^{2,3}$, Pascal Niamba ${ }^{2,3}$ \\ ${ }^{1}$ Department of Dermatology, Raoul Follereau Center, Ouagadougou, Burkina Faso, ${ }^{2}$ University Ouaga I Professor \\ Joseph Ki-Zerbo, Ouagadougou, Burkina Faso, ${ }^{3}$ Department of Dermatology, University Hospital Yalgado Ouédraogo, \\ Ouagadougou, Burkina Faso, ${ }^{4}$ Department of Dermatology, Regional Hospital of Ouahigouya, Ouahigouya, Burkina Faso
}

Corresponding author: Dr. Amina Nomtondo Ouedraogo, E-mail: Nomtondo2000@yahoo.fr

\begin{abstract}
RÉSUMÉ
Introduction: Lacné est une maladie fréquente. Sa prévalence atteint $80 \%$ chez les adolescents dans le monde. A Ouagadougou, elle était de 57,1\% en 2001 en milieu scolaire. La littérature médicale est très fournie sur les aspects épidémiologiques, cliniques et thérapeutiques de l'acné, et l'est moins sur les perceptions et connaissances des patients souffrant d'acné. Lobjectif de cette etude était d'évaluer la prévalence, les connaissances, les croyances, des élèves et étudiants de la ville de Ouagadougou sur l'acné. Méthodes et Patients: Nous avons réalisé une étude transversale de juin à juillet 2014 chez les étudiants de l'université de Ouagadougou et les élèves de 4 établissements secondaires. Résultats: Nous avons enquêté 425 personnes dont 40,6\% de filles et 59,4\% de garcons. La prévalence de l'acné était de 54,8\%. Lâge moyen était 19,53 ans, avec des extrêmes allant de 13 ans et 30ans. Parmi les etiologies de l'acné, l'alimentation était citée par $40,05 \%$ des enquêtés, la puberté par 25,43\%, les cosmétiques par 14,03\%. Parmi les aliments en cause, les arachides ont été cités par $37,96 \%$, la mayonnaise par $21,22 \%$, le beurre par $21,52 \%$, les sucreries par 8,07\% et le chocolat par 9,41\%. L’huile était incriminée par 72,6\% et le sucre 5,5\%. Le tabac était cité par 19,70\% comme facteur favorisant, l'absence de rapport sexuels par 19\%. Parmi les 192 sujets non acnéiques, 17\% liaient l'acné à un manque d'hygiène, $80 \%$ seraient gênés d'avoir un partenaire acnéique, Les cosmétiques soigneraient l'acné pour $37,90 \%$ et les produits naturels pour 6,30\%. Ceux qui ont eu recours à un dermatologue étaient 15,02\%. Conclusion: Les élèves et étudiants connaissent mal l'acné et perçoivent mal leurs camarades acnéiques.
\end{abstract}

Mots Clés: Alimentation; Étiologie acné; Connaissance

\section{INTRODUCTION}

Lacné est une pathologie inflammatoire du follicule pilo-sébacé fréquente, dont la prévalence atteint $80 \%$ chez les adolescents dans le monde et $40 \%$ chez les adultes [1-3]. Sa prévalence en milieu scolaire à Ouagadougou était de 57,1\% en 2001 [4].La littérature médicale est très fournie sur les aspects épidémiologiques, cliniques et thérapeutiques de l'acné $[1,2,5-7]$, mais il en existe moins sur les perceptions, la connaissance et la compréhension des patients de leur maladie.

Au Burkina Faso, aucune étude n'est encore disponible sur ce sujet. Avoir de telles données pourrait être utile à l'amélioration de la relation médecin-malade dans la prise en charge de l'acné, à l'élaboration d'outils, de programmes d'information de communication et de sensibilisation adaptés aux besoins spécifiques des jeunes.Lobjectif de cette étude est d'évaluer

\footnotetext{
How to cite this article: Ouedraogo AN, Kabre Ouedraogo SSS, Ouedraogo MS, Traore F, Tapsoba PG, Barro-Traore F, Traore A, Niamba P. Acné: prévalence, perceptions et croyances chez les élèves et étudiants à Ouagadougou, Burkina Faso. Our Dermatol Online. 2017;8(1):10-16.

Submission: 13.10 .2016 ; Acceptance: 21.11 .2016

DOI: $10.7241 /$ ourd.20171.03
} 
la prévalence, les connaissances, les croyances, et perception des élèves et étudiants de la ville de Ouagadougou sur l'acné.

\section{MATERIALS AND METHODS}

\section{Type d'étude, Lieu et Période}

Notre étude était de type transversale descriptive. Elle s'est déroulée dans deux Unités de Formation et de Recherche (UFR) (UFR Sciences Juridiques et Politques, et UFR Sciences Economiques et de Gestion) de l'université de Ouagadougou (devenue université Ouaga I Professeur Joseph Ki-Zerbo) et dans quatre centres d'examen du Baccalauréat (BAC) et du Brevet d'Etude du Premier Cycle (BEPC) des établissements secondaires de la ville de Ouagadougou(Lycée Philipe Zinda Kaboré, Lycée Nelson Mandela, Lycée Marien Ngouabi, Collège notre Dame de Kologh Naba) de juin à juillet 2014.

\section{Echantillonnage}

La population d'étude était choisie de manière raisonnée compte tenu de nos ressources humaines, et financières limitées. Nous avons retenus les étudiants de l'université et les élèves candidats aux examens du BEPC et du BAC de la ville de Ouagadougou au cours de l'année scolaire 2013-2014 comme population d'étude. La taille minimale $\mathrm{N}$ de l'échantillon a été calculée suivant la formule suivante: $\mathrm{N}=\left[\mathrm{E}^{2} \cdot \mathrm{P} \cdot \mathrm{Q}\right] / \mathrm{i}^{2}$ $\mathrm{N}=$ taille minimale de l'échantillon $\mathrm{E}=$ écart réduit pour le risque $\alpha=5 \%$ (loi normale); une précision de $6 \%$ avec un risque d'erreur $\alpha=5 \%$. Sa valeur était de $1,96 \mathrm{P}=57,1 \%$ (prévalence acné milieu scolaire en 2001) [4]; $\mathrm{Q}=1-\mathrm{P}-\mathrm{i}=$ précision désirée; soit $\mathrm{i}=5 \%$. $\mathrm{N}$ ajusté à $10 \%$ était 425 .

Les étudiants de l'université de Ouagadougou étaient au nombre de 22.808 au cours de l'année 2013-2014. Pour le choix de l'échantillon d'étudiants,le premier degré du sondage a consisté à un tirage aléatoire de deux UFR parmi les 6 que compte l'université. Le deuxième niveau au tirage aléatoire de 57 étudiants dans chacune des 2 UFR sélectionnée soit 114 étudiants au total.

Pour le choix du nombre d'élèves, nous avons fait un choix proportionnel au nombre de candidats par niveau d'examen. La ville de Ouagadougou comptait pour l'année scolaire 2013-2014, 61668 élèves, candidats aux examens du BAC et du BEPC, dont 41005 candidats à l'examen du BEPC et 20663 candidats pour le BAC. Nous avons retenu donc 208 candidats du BEPC et 103 candidats du BAC proportionnellement à la taille de la population d'étude. Pour le choix des jurys et des élèves, la technique de sondage en grappe a été utilisée. Le premier degré du sondage a consisté au tirage aléatoire de deux centres d'examen du BEPC, puis le second niveau du sondage au tirage de 208 élèves dans ces centres. Nous avons procédé de même, pour le tirage de 103 élèves candidats au BAC.

Les élèves et étudiants ont été enrôlés dans l'étude au fur et à mesure qu'ils étaient rencontrés dans la cour de l'établissement jusqu'à l'atteinte du nombre voulu. Ils ont été interrogés dans un local aménagé à cet effet.

\section{Critères D'inclusion}

Ont été inclus les étudiants des UFR retenues et les élèves candidats aux examens du BEPC ou du BAC, des centres d'examens retenus ayant donné leur consentement à participer à l'étude.

\section{Méthode et Recueil des données}

Le recueil des données était fait par un questionnaire comprenant les éléments sociodémographiques. Des questions ouvertes, semiouvertes ont été utilisées pour tester les connaissances et croyances et pratiques des élèves et étudiants.

\section{Analyse Statistique}

Le test de Student était utilisé pour la comparaison des moyennes et le Khi-deux pour l'indépendance et les liaisons entre les données statistiques. Une valeur seuil $\mathrm{p}<5 \%$ était retenu comme significatif.

\section{Ethics Statement}

Cette étude a obtenu un avis favorable du ministère des enseignements secondaire supérieur et de la recherche scientifique. Les entretiens ont été menés en respectant la dignité des élèves, l'anonymat était assuré dans le traitement des données.

\section{RESULTS}

Nous avons enquêtés 425 personnes dont $40,6 \%$ de filles et les garçons $59,4 \%$. La prévalence globale de l'acné était de 54,8\%.Chez les sujets de sexe féminin, elle était de $55,90 \%$ et $53,10 \%$ chez ceux de sexe masculin. 
Lâge moyen était 19,53 ans avec des extrêmes allant de 13 ans et 30 ans

A la question ouverte de savoir quelles etaient les étiologies de l'acné ? Lalimentation, la puberté, les cosmétiques venaient en tête des réponses, $40,05 \%$, $25,43 \%$ et $14,03 \%$ respectivement. Les hormones, le stress, la séborrhée ont été cités respectivement par $3,21 \%, 2,33 \%$ et $0,87 \%$. The Figure 1 nous donne l'exhaustivité des réponses.

A la question de savoir lequel des aliments cités (arachide, chocolat, sucrerie, mayonnaise, beurre, autre) pouvaient favoriser l'acné, les arachides ont été cités par 37,96\%, la mayonnaise et le beurre respectivement $21,22 \%, 21,52 \%$, les sucreries et le chocolat $8,07 \%$ et $9,41 \%$ et autre $1,79 \%$.

Parmi les 274 enquêtés ayant incriminés les aliments dans la survenue de l'acné,72,6\% (199) des personnes pensaient que l'huile contenue dans les aliments était responsable de la survenue de l'acné et 5,5\% (15) pensaient que c'était plutôt le sucre contenu dans l'aliment qui en était responsable. Lallergie à ces aliments déclencherait l'acné pour $12 \%$ (33), et 9,9\% (27) évoquaient leur expérience vécu.

A la question de savoir si les éléments cités (tabac, stress, menstrues, absence de rapport sexuel, autre) étaient des facteurs favorisants l'acné,40,90\%ont cités les menstrues, $30,80 \%$ le stress, $19,70 \%$ le tabac, $19 \%$ l'absence de rapport sexuels et $2,4 \%$ ont répondus autres. La comparaison entre les réponses des élèves et celles des étudiants pour les causes de l'acné, n'a pas noté de différence significative.

Parmis les 192 sujets non acnéiques interrogés, 17\% pensaient que l'acné était lié à un manque d'hygiène, $40 \%$ pensaient qu'il s'agissait d'une maladie, $37 \%$ d'un phénomène physiologique normal et $6 \%$ ont donné diverse autre réponse. Parmi les sujets non acnéiques, $80 \%$ seraient gênés d'avoir un partenaire souffrant d'acné (dont 24\% beaucoup gênés), 20\% ne seraient pas du tout gênés, $74.40 \%$ pensaient que l'acné altérait l'image de soi et 25,6\% ne le pensaient pas.

26,11\% (111/425) pensaient que l'acné était incurable $1,41 \%$ (6/425) n'avaient aucune idée sur les possibilités de traitement de l'acné, 55,70\% (141/425) pensaient que l'acné pouvait être soignée par les produits pharmaceutiques dont ceux contenant du peroxyde de benzoyle, des rétinoïdes topiques et antibiotiques topiques. Les cosmétiques (savon, crème et lotion) ont été cités par 37,90\% (96/425) comme suffisant pour soigner l'acné. Les produits naturels tel le miel, le citron, l'aloe vera, l'huile de neem par 6,30\% (16/425). Parmi les sujets souffrants d'acné, 15,02\% (35/233) avaient eu recours eu recours à un dermatologue pour traiter leur acne.

\section{DISCUSSION}

Lacné est une maladie fréquente, si certains auteurs rapportent des prévalences de 80 à $90 \%$ [1,3], notre étude trouve $54,8 \%$ chez les élèves et étudiants de la ville de Ouagadougou. La prévalence est plus importante chez les filles 55,90\% que chez les garçons $53,10 \%$. Cette tendance est généralement retrouvée dans la littérature. Uslu en Turquie [8] dans son étude intitulée, Acné: prévalence, perceptions et effets sur la santé psychologique chez les adolescents à Aydin avait rapporté une prévalence de $63,6 \%, 53,8 \%$ chez les filles et $46,2 \%$ chez les garçons. L'âge moyen des enquêtés de Uslu était de 15,24 ans tandis que nous trouvions 19,53 ans. Cette différence s'explique par le site de recrutement de notre série en milieu universitaire et le second cycle du lycée.

Lacné est une maladie multifactorielle. Parmi les éléments en cause, l'augmentation de la production de sébum, l'hyperkératinisation infundibulaire, la prolifération de Propionibacterium acnes et les facteurs de l'inflammation sont admis dans sa physiopathogénie $[9,10]$. D’une manière générale, les élèves et étudiants ne connaissent pas les causes de l'acné. La majorité des enquêtés ont plutôt cités l'alimentation $(40,05 \%)$ et la puberté $(25,43 \%)$ comme principales étiologies de l'acné. Dans le contexte africain en générale et burkinabé en particulier, les boutons d'acné sont très couramment appelés «boutons d'adolescence » [11], 37\% des sujets non acnéiques pensaient que l'acné est un phénomène physiologique normal. Ceci pourrait également expliquer le lien fait entre la puberté et l'acné par nos répondants. Ce qui semble justifier car l'acné débute généralement à la puberté avec l'afflux d'hormones notamment la testostérone [12].

L'hyperséborrhée, le stress, les hormones bien documentés dans la physiopathologie de l'acné [12] ne sont cités que par une petite minorité de nos enquêtés, respectivement $0,87 \%, 2,33 \%$, et $3,21 \%$. Pourtant l'implication du stress dans la survenue de l'acné a 
été prouvée [9]. Des cellules nerveuses situées près de la glande sébacée peuvent produire une substance, dite substance $\mathrm{P}$, libérée sous l'effet du stress qui peut stimuler la production de sébum [9]. Tan et al. [13] ont rapporté que $71 \%$ de leurs patients considéraient le stress comme un facteur important dans la survenue de l'acné. Une explication possible est la sécrétion d'androgène surrénale pendant les périodes de stress et les effets subséquents sur l'hyperactivité sébacée.

Les produits cosmétiques cités par 14,03\% de nos répondants et les produits dépigmentants par 2,92\% sont également incriminés dans la survenue de l'acné. Ces réponses ont probablement été motivées par le vécu et l'expérience personnelle des enquêtés ou de leur entourage. En effet, le phénomène de la dépigmentation prend de l'ampleur en Afrique de l'Ouest et particulièrement au Burkina. Traoré et al [14] trouvaient déjà en 2005, 44,3\% de femmes qui pratiquaient régulièrement la dépigmentation artificielle à Ouagadougou. Les élèves et étudiants représentaient 32,5\% des sujets interrogés. Lacné était comptée parmi les complications dermatologiques retrouvées chez $58 \%$ de l'échantillon.

Nos répondants ont incriminé l'alimentation dans la survenue de l'acné à 40,05\%, notamment les aliments à index lipidique élevé tel l'arachide $(37,96 \%)$, le beurre $(21,52 \%)$, la mayonnaise $(21,22 \%)$. Ces aliments ont été cités sur la base d'observation faite sur le vécu, comme l'indiquait cette phrase citée par une élève «Chaque fois que je mange de l'arachide ou du beurre, le lendemain, j’ai des boutons sur le visage ». Les sucreries et le chocolat ont été moins cités, cela serait en rapport avec les habitudes alimentaires du citoyen burkinabé «lambda » incluant peu le chocolat. Nos résultats se rapprochent des constatations de Peiqi Su à Singapore [15] dans une étude sur les croyances, perceptions et impact psychosocial de l'acné parmi les étudiants d'un institut tertiaire. Il a rapporté que l'alimentation était vue par 38,7\% comme un facteur aggravant de l'acné notamment les aliments épicés et les fritures. Quant à Fekih, Di Landro, Burris, ils ont souligné l'implication des aliments à index glycémique élevé dans les poussées acnéiques [16-18]. Ces aliments accroissent la production d'insuline qui contribuait à la formation d'hormones androgéniques favorisant la production de sébum donc l'apparition de l'acné. Pourtant, Smith [19] a rapporté que la consommation hebdomadaire de gâteaux, de bonbons et de chocolat n'a pas été associée à un risque plus élevé de l'acné. Le

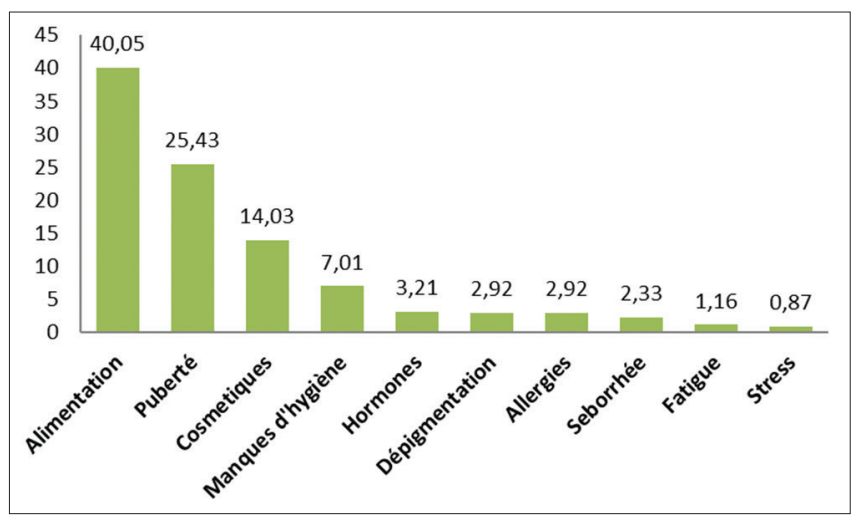

Figure 1: Répartition des étiologies en pourcentage.

rôle de l'alimentation dans l'acné reste donc d'actualité et controversé et des recherches plus poussées sur le sujet doivent se poursuivre.

Le tabac cité par 19,70\% de nos enquêtés serait un facteur favorisant la survenue de l'acné. Cependant, certaines études réfutent l'implication du tabac dans la survenue de l'acné et d'autres l'acceptent. En effet, Peiqi Su [15], a rapporté que $18.9 \%$ des sujets croient que l'acné a un lien avec la consommation de tabac. Capitanio [20] a positivement corrélée l'acné au tabagisme par le nombre de cigarettes quotidiennement fumés et la prévalence de l'acné d'apparition tardive. T. Chuh et col [21], dans une étude réalisée à Hong Kong et en Inde, l'association du tabagisme à l'acné chez les hommes a rapporté une augmentation de la sévérité de l'acné significativement associée à une augmentation du stress. Plus, les gens étaient stressés plus ils fumaient, le stress pourrait donc constitué un facteur de confusion dans l'association acné et tabagisme.

La comparaison entre les réponses des élèves et celles des étudiants sur les causes de l'acné n'a pas noté de différence significative. Ce qui nous laisse penser que le niveau d'étude n'influe pas sur le niveau de connaissance sur l'acné des élèves et étudiants et que les perceptions populaires et culturelles de la maladie prédominent. Cependant, il faut préciser que l'échantillon a regroupé des étudiants des facultés de sciences politiques et juridiques, ainsi que des sciences économiques et de gestion. Les réponses auraient probablement varié si l'échantillon avait prit en compte des étudiants en médecine ou pharmacie.

Parmi les 45,2\% de sujets ne souffrant pas d'acné, $17 \%$ reliaient l'acné à un manque d'hygiène, percevant ainsi à tort leur camarade acnéique comme une 
personne sale et $19 \%$ à une absence de relation sexuelle. Aussi, nous pouvons comprendre pourquoi $80 \%$ des non acnéiques déclaraient être gênés si leur partenaire avait de l'acné; et 74,4\% d'entre eux étaient convaincus que l'acné diminuait la beauté. Ceci pourrait accroitre le mal-être du sujet acnéique dans son milieu, favoriser son isolement social ou l'entrainer à avoir des comportements sexuels à risque dans le but de faire disparaître les lésions d'acné. Carol E cheng et al au Etats Unis [22], dans leur étude sur la gravité de l'acné, le traitement et modèles de croyance à travers les groupes raciaux et ethniques multiples des élèves adolescents ont aussi noté que 77\% trouvent que l'acné rend les personnes atteintes moins attrayantes. Cette fausse idée associant l'acné à un manque d'hygiène était également rapportée par des auteurs asiatiques. Peiqi Su [15] notait que 65\% des répondants liaient l'acné à une mauvaise hygiène personnelle. Suh a révélé que $48,4 \%$ de sujets ont estimé que leur acné s'empirait s'ils ne se lavaient pas le visage à fond tandis que $10 \%$ estiment que leur acné s'améliorait s'ils ne se lavaient pas le visage. Tan a également rapporté que $62 \%$ de son échantillon considéraient la saleté comme un facteur favorisant important de l'acné [23].

Face à leur acné, 15,02\% seulement des sujets acnéiques ont recours à l'expertise d'un dermatologue pour se soigner, le reste préférant se soigner par d'autres moyens en ayant recours à des cosmétiques dans $37,90 \%$ ou des produits naturels tel le citron le miel, l'aloe vera dans 6,30\%. D'ailleurs 26,11\% des sujets souffrant d'acné la croient incurable. Ce qui est justifié par le contexte culturelle et économique. Le traitement classique d'une acné légère à modérée est suspensif, et nous avons noté une difficulté de la population illettrée (majorité de la population burkinabé) à comprendre la notion de maladie inflammatoire chronique. Lun des traitements susceptibles de donner des résultats définitifs en interrompant les poussées, en l'occurrence l'isotrétinoïne est hors de portée pour la majorité des patients burkinabés moyens ne disposant pas d'assurance maladie. Néanmoins $55 \%$ ont pu citer les produits pharmaceutiques adaptés (peroxyde de Benzoyle, rétinoïde topique, antibiotique topique) au traitement topique de l'acné. Uslu [8] notait une perception différente chez les adolescents de son étude. Moins de la moitié considèrent l'acné comme une «maladie», et la plupart d'entre eux pensaient qu'elle est curable et que son traitement était une nécessité.

\section{CONCLUSION}

Malgré la prévalence élevée de l'acné chez les jeunes scolarisés de la ville de Ouagadougou, il y a un déficit de connaissance sur les causes, les facteurs favorisants, le traitement de la maladie. Peu consultent les spécialistes pour se faire prendre en charge. Envisager un programme d'information, d'éducation et de sensibilisation (IEC) à l'endroit des élèves et étudiants pourrait leur donner une meilleure connaissance et compréhension de l'acné, leur permettre de recourir aux soins des professionnels de la santé et influencer positivement leurs comportements face à leur acné ou celle de leur camarade.

\section{REFERENCES}

1. Dréno B. Données récentes sur l'épidémiologie de l'acné. Annal Dermatol Vénéréol. 2010;137:S49-S51.

2. Schäfer T, Nienhaus A, Vieluf D, Berger J, Ring J. Epidemiology of acne in the general population: the risk of smoking. Br J Dermatol. 2001;145:100-4.

3. Dreno B, Poli F. Epidemiology of acne. Dermatology. 2003;206:7-10.

4. Traore A, Ouedraogo MS, Niamba P, Barro-Traoré F, Drabo YJ. Aspects epidemiologiques et cliniques de l'acne en milieu scolaire de la ville de Ouagadougou (Burkina Faso). Annal Dermatol Venereol. 2001;134:165.

5. Seité S, Rougier A, Dréno B. Enquête sur la prise en charge des patients acnéiques en France. Annal Dermatol Vénéréol. 2012;139:611-6.

6. Rzany B, Kahl C. Epidemiology of acne vulgaris. J Dtsch Dermatol Ges. 2006;4:8-9.

7. Dreno B, Bodokh I, Chivot M, Daniel F, Humbert P, Poli F, et al. ECLA grading: a system of acne classification for every day dermatological practice.. Annal Dermatol Venereologie. 1999;126:136-41.

8. Uslu G, Sendur N, Uslu M, Savk E, Karaman G, Eskin M. Acne: prevalence, perceptions and effects on psychological health among adolescents in Aydin, Turkey. J Eur Acad Dermatol Venereol. 2008;22:462-9.

9. Vexiau P, Chivot M. [Feminine acne: dermatologic disease or endocrine disease?]. Gynecol Obstet Fertil. 2002;30:11-21.

10. Drapier-Faure E, Faure M. Quelle est la place des traitements hormonaux dans l'acné? Ann Dermatol Venereol. 2001;128:S19-24.

11. Kouotou EA, Adegbidi H, Belembe RB, Sieleunou I, Nansseu JR, Kamga JP, et al. [Acne in Cameroon: Quality of life and psychiatric comorbidities]. Annal Dermatol Venerol. 2016;143:601-6.

12. Dreno B. Acné. EMC - Dermatol-Cosmetol. 2002;98:11.

13. Tan JK, Vasey K, Fung KY. Beliefs and perceptions of patients with acne. J Am Acad Dermatol. 2001;44:439-45.

14. Traore A, Kadeba J-C, Niamba P, Barro F, Ouedraogo L. Use of cutaneous depigmenting products by women in two towns in Burkina Faso: epidemiologic data, motivations, products and side effects. Int J Dermatol. 2005;44:30-2.

15. Su P, Chen Wee Aw D, Lee SH, Han Sim Toh MP. J Dtsch Dermatol Ges. 2015;13:227-33.

16. Fekih N, Aounallah-Skhiri H, Hajlaoui K, Kamoun R. Évaluation du rôle de l'alimentation dans la survenue de l'acné de l'adolescent. Annal Dermatol Vénéréol. 2011;12:A165.

17. Di Landro A, Cazzaniga S, Parazzini F, Ingordo V, Cusano F, Atzori L, et al. Family history, body mass index, selected dietary 


\section{www.odermatol.com}

factors, menstrual history, and risk of moderate to severe acne in adolescents and young adults. J Am Acad Dermatol. 2012;67:1129-35.

18. Burris J, Rietkerk W, Woolf K. Acne: the role of medical nutrition therapy. J Acad Nutr Diet. 2013;113:416-30.

19. Smith RN, Mann NJ, Braue A, Mäkeläinen H, Varigos GA. A lowglycemic-load diet improves symptoms in acne vulgaris patients: a randomized controlled trial. Am J Clin Nutr. 2007;86:107-15.

20. Capitanio B, Sinagra JL, Ottaviani M, Bordignon V, Amantea A, Picardo M. Acne and smoking. Dermatoendocrinol. 2009;1:129-35.

21. Chuh AAT, Zawar V, Wong WCW, Lee A. The association of smoking and acne in men in Hong Kong and in India: a retrospective case-control study in primary care settings. Clin Exp Dermatol. 2004:29:597-9.
22. Cheng CE, Irwin B, Mauriello D, Liang L, Pappert A, Kimball AB. Self-Reported Acne Severity, Treatment, and Belief Patterns across Multiple Racial and Ethnic Groups in Adolescent Students. Pediatr Dermatol. 2010;27:446-52.

23. Tan JK. Psychosocial impact of acne vulgaris: evaluating the evidence. Skin Ther Lett. 2004;9:1-3.

Copyright by Amina Nomtondo Ouedraogo, et al. This is an open-access article distributed under the terms of the Creative Commons Attribution License, which permits unrestricted use, distribution, and reproduction in any medium, provided the original author and source are credited.

Source of Support: Nil, Conflict of Interest: None declared. 